\title{
Development of Algorithms for Digital Control for Mechatronic Drives Consisting of Medical Devices for Rehabilitation
}

\author{
E. Novikova ${ }^{1}$, V. Morozov ${ }^{1}$, and A. Zhdanov ${ }^{1}$ \\ ${ }^{1}$ Vladimir State University named after Alexander and Nikolay Stoletovs, Russian Federation.
}

Keywords: Mathematical model, mechatronic drive, algorithm, microprocessor control system.

\begin{abstract}
The dynamics of a mechatronic drive is investigated based on a dual-mass mathematical model. To obtain a closed, fully defined model of movement of the biomechanical trainer during the development we should determine the law of control with the mechatronic drive of the BMT. In the article The article considers development of algorithms for digital control for mechatronic drives consisting of medical devices for rehabilitation.
\end{abstract}

\section{Introduction}

The dynamics of a mechatronic drive is investigated based on a dual-mass mathematical model. Since in a monoblock structure drive of the actuator is placed in a hollow rotor of the electric machine, we will take for the first mass an input segment of the PC, integrated in the rotor, but for the second, we will take the output segment that translates the load linearly.

\section{Mathematical Model of the Dynamics of the Drive}

The equation of the mechanical characteristic of the direct current motor has the form [1]

$$
\omega_{1} / \omega_{x x}+M_{1} / M_{\Pi}=U_{y n p} / U_{0}
$$

where $\omega_{1}$ is the angular velocity of rotation of the first segment; $\omega_{X X}$ is the angular velocity of the ideal idle; $M_{1}$ is the torque on the motor's shaft; $M_{\Pi}$ is the starting torque of the motor; $U_{y п p}$ is the voltage at the input of the motor; $U_{0}$ is the rated motor's voltage.

The angular velocity of rotation of the first segment is

$$
\omega_{1}=u \omega_{2}
$$

where $\omega_{2}=\varphi_{2}$ is the angular velocity of rotation of the second segment (the load); $u$ is the kinematic velocity ratio of the $\mathrm{PC}$.

The torque on the motor's shaft is defined as the sum of the moment of inertia of the motor's shaft and the load moment to the motor's shaft

$$
M_{1}=\left(J_{1}+J_{\text {рот }}\right) \dot{\omega}_{1}+M_{2}^{\text {пр }}
$$

where $J_{1}$ is the moment of inertia of the input segment; $J_{\text {pom }}$ is the moment of inertia of the engine's rotor; $M_{2}^{\Pi p}$ is the modified load moment:

$$
M_{2}^{\text {пр }}=1 / u \eta\left(J_{2} \dot{\omega}_{2}+M_{2}\right),
$$

where $\eta$ is the coefficient of efficiency of the PC; $J_{2}$ is the moment of inertia of the output segment; $M_{2}$ is the load moment.

Taking into account the expressions (2) - (4), the equation (1) takes the form 


$$
u \omega_{2} / \omega_{\mathrm{xx}}+\left[u\left(J_{1}+J_{\text {рот }}\right)+\frac{1}{u \eta} J_{2}\right] \dot{\omega}_{2}+\frac{1}{u \eta} M_{2} / M_{\text {п }}=U_{\text {упр }} / U_{0}
$$

Converting the differential equation (5) to a normal form relative to the angular velocity $\omega_{2}$, we have:

$$
T_{\gamma} \dot{\omega}_{2}=\omega_{u}\left(U_{\text {упр }} / U_{0}-1 / u \eta M_{2} / M_{\text {п }}\right)-\omega_{2},
$$

where $T_{\gamma}=\left(J_{1}+J_{\text {рот }}+1 / u^{2} \eta J_{2}\right) \omega_{\mathrm{xx}} / M_{\Pi}$ is the electromechanical constant of the time of the input segment of the drive; $\omega_{u}=\omega_{\mathrm{XX}} / u$ is the angular velocity of the ideal idle, modified to the output segment.

The load moment in the joint $M_{2}$ depends on the magnitude of the angle extension $\varphi=\varphi 2$, of the myotonus and the contraction index by the power law:

$$
M_{2}=M_{0}+M_{\text {пр }}\left(\varphi / \varphi_{\text {пр }}\right)^{\frac{1}{k}},
$$

where $M_{0}$ - is the moment of the resistance caused by the initial myotonus of the flexors; $М п p$ is the moment of the resistance at full extension of the joint at the angle $\varphi п p ; k$ is the index of the contracture.

Contracture index $k$ varies from 0 to 1 . The higher the score, the less joint mobility, which is limited by a threshold value of the load moment $M \kappa p$. The critical value of the $M \kappa p$ corresponds to the load moment, above which comes during the phase of contraction (for example, the painful sensations arise). Fig. 1 shows the dependence of the load moment on the extension angle with different indices of contracture through the example of the elbow joint (for the initial position was taken a position with a bent angle of $90^{\circ}$ ). For this model when the index $k=0,1$ the amplitude of the joint mobility is $56,2^{\circ}$, when $k=0,05$ is $71,1^{\circ}$, when $k=0,01$ is $85,9^{\circ}$, when $k=0,001$ is $89,6^{\circ}[2]$.

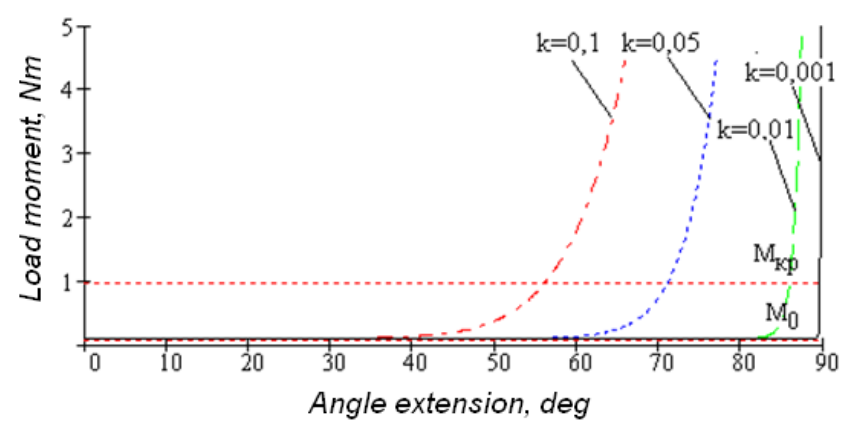

Fig. 1. The law of the variation of the load moment at different values of the index of contracture.

We notice that the index of contracture is not a constant parameter, but it decreases during the development process. To formalize of this dependence we should have a sufficiently large amount of clinical data, moreover, this dependence is, apparently, individual for each patient.

The system of equations of the dynamics of the drive of the biomechanical trainer has the form [3]:

$$
\left\{\begin{array}{l}
\dot{\varphi}_{2}=\omega_{2} ; \\
\dot{\omega}_{2}=\omega_{u}\left(U_{\text {упр }} / U_{0}-1 / u \eta M_{2}\left(\varphi_{2}\right) / M_{\Pi}\right) \omega_{2} / T_{\gamma},
\end{array}\right.
$$

where $M_{2}$ is changed according to the law (7).

To obtain a closed, fully defined model of movement of the biomechanical trainer during the development we should determine the law of control with the mechatronic drive of the BMT. 


\section{Synthesis of the Algorithm of the Digital Control with Drives Consisting of BMT}

In the drive, the algorithm of control is implemented by the motion of the articulated-lever system of the trainer for the development of contractures with a decrease of the painful sensations.

The action of the algorithm is based on the registration of the magnitudes of the moment of resistance. The drive accomplishes the extension movements in the joint as long as the resistance does not reaches the threshold value $М \kappa p$. In this case, the drive switches to an oscillatory mode, forcing the joint to accomplish rapid oscillatory movements of small amplitude. This mode helps to relax the flexor muscles and reduce the painful sensations. The oscillating mode operates for a certain period of time (from several seconds to several minutes), which is given programmatically, then the actuator returns to its original position, forcing the joint to bend and giving the opportunity to restore the local blood circulation and the relaxation of the extensor. Then the cycle is repeated again. [4].

The scheme of work of the algorithm in the course of one development cycle is shown in the Fig. 2

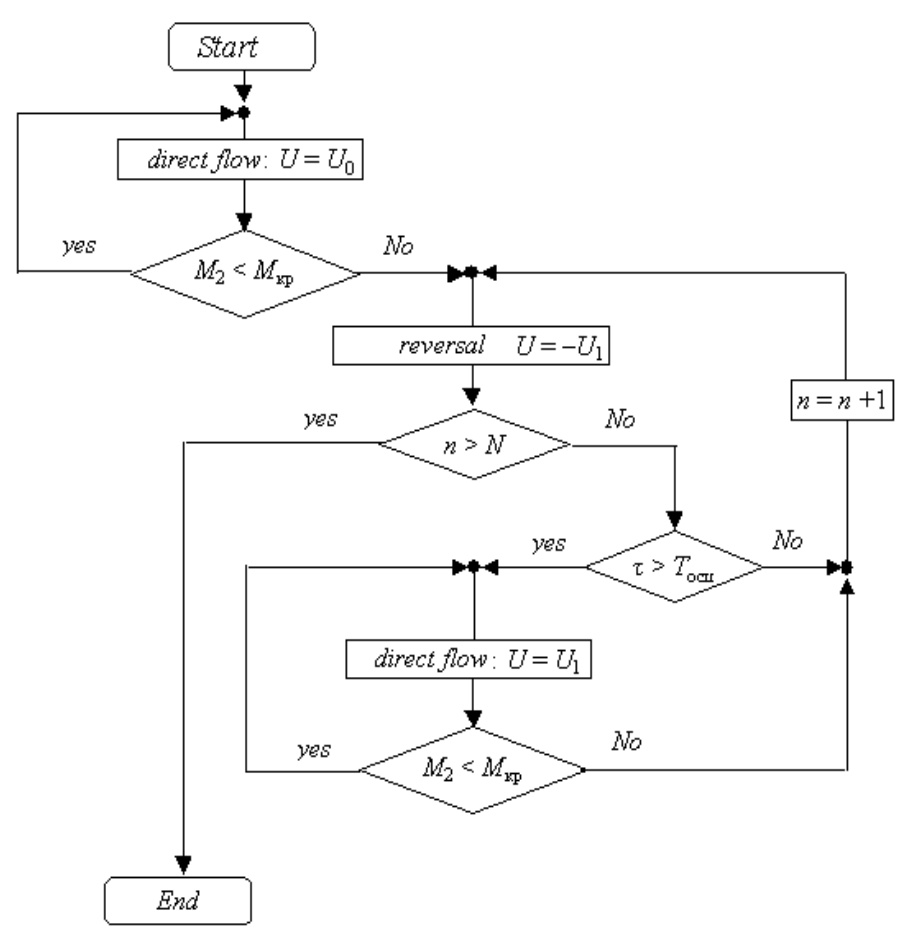

Fig. 2. Scheme of the algorithm in the course of one development cycle.

The algorithm works as follows. Under the influence of the rated voltage $U=U_{0}$ the drive accomplish the extension of the joint (straight lead) as long as the resistance reaches a critical value of the Мкр. This torque is fixed by the current sensor, because the consumption current is directly proportional to the overcome load:

$$
I=M_{2} / c_{M},
$$

where $M$ is the relation of the static synchronizing torque of the motor to the starting current of the phase.

When the achievement $M_{2}=M \kappa p$ switches to a mode of oscillation. The drive under the influence of the control $U=-U_{1}$ starts working off the movement in reverse (bending of the joint) during a given half-cycle of oscillation Тосц. The amplitude of the control voltage $U_{1}$ is determined from the passage condition of a specified extension angle $\alpha$ for a half-cycle:

$$
U_{1}=2 \pi \alpha / \omega_{u} T_{\text {осц }} \sqrt{1+\left(2 \pi T_{\gamma} / T_{\text {осц }}\right)^{2}} U_{0} .
$$


At the end of the half-cycle at the time $\tau=$ Tосц switches the control to an straight lead $U=U_{1}$, forcing the joint to straighten oneself up. The extension continues as long as the event occurs again $M_{2}=M \kappa p$, then a second oscillations cycle is accomplished.

During each vibration in the oscillation phase, the counter of cycles $\mathrm{n}$ records the number of the oscillations accomplished. During the accomplishment of a predetermined number $N$ of oscillations, the phase of oscillations ends, the drive returns to its original position under the influence of the control voltage $U=-U_{1}$ and the development cycle ends.

For the working algorithm it is necessary to specify the following parameters:

$M \kappa p$-is the threshold value of the torque of the resistance, that characterizes the onset of contractures, $\mathrm{N} \cdot \mathrm{m}$;

$M$-is the number of cycles of development during the session of development;

$N$-the number of oscillations during the development cycle;

Tосц-is the half-cycle of an oscillation, $s$;

$\alpha$ - is the amplitude of oscillation, degree.

The rest of the parameters (engine characteristics: $\omega_{X X}, M_{\Pi}, J_{p o T}, U_{0}$; reducer: $u, \eta, J_{1}, J_{2}$; extreme angle of extension of the joint $\varphi п p$ ) are fixed and are sewn into the ROM of the microprocessor. They can also be reprogrammed, for example, during the realignment of the trainer to other joint, during the substitution of the motor or the reductor, but in contrast to the above enumerated parameters of control, they are not available via user console [2].

\section{Simulation Modelling of the Dynamics of the BMT}

To test the efficiency of the proposed algorithm, a complex of research was led according to the simulation modelling of the dynamics of the trainer.

We notice that does not matter the exactly form of the dependence (7) for the algorithm and specially the value of the index of contracture (which is essentially tied to the proposed model). But for the simulation model it is necessary a formalized description of the efforts of resistance of extension depending on the angle of extension, taking into account the positive dynamics in the development process.

The Fig. 3-Fig. 7 show the results of the simulation modelling: diagrams of the change in the angle and angular velocity of the extension, the control voltage, the torque of resistance and the index of contraction for of the development cycle. For using of visual methods, was proposed an idealized case, when each oscillation was effective - after each oscillation the exponent $1 / \mathrm{k}$ in the expression (7) is incremented by one. The mode of oscillation is TocЦ $=0,1 c, \alpha=20^{\circ}$. The mobility in the joint before the development was $56,1^{\circ}$, at the end of the development cycle was $80,1^{\circ}$. It can be seen that the reverse lead begins at the moment when $M_{2}=$ Mкp [5].

The straight lead during each oscillation is accomplished for a longer time than the reverse. This is due to the fact that during the straight lead, the drive has to overcome the resistance, whereas during the reverse lead of the load helps the trainer to bend the joint.

The main result of the simulation modelling is to test the working capacity of the algorithm. The built implementation in the diagrams confirms that the algorithm provides the required oscellation mode.

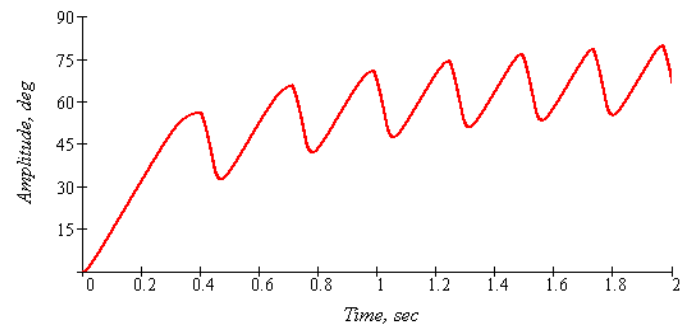

Fig. 3. Diagram of the change in the extension angle for a development cycle. 


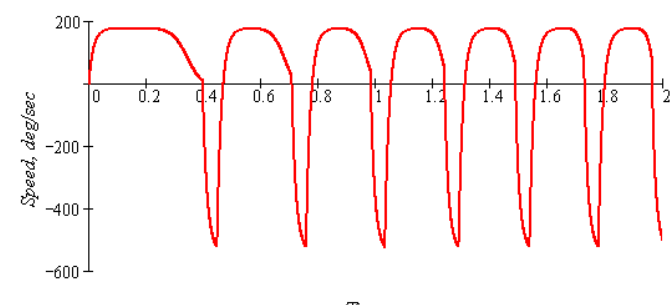

Fig. 4. Diagram of the change in the angular velocity of the extension for a development cycle.

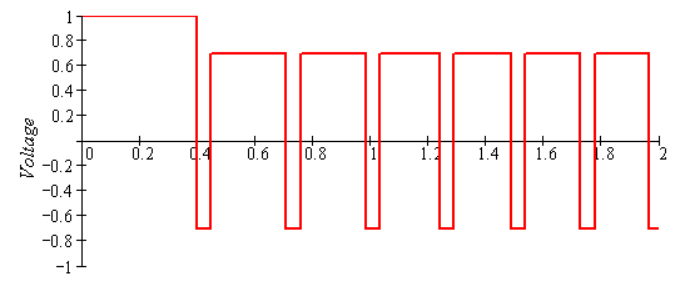

Time, sec

Fig. 5. Diagram of the change in the control voltage for a development cycle.

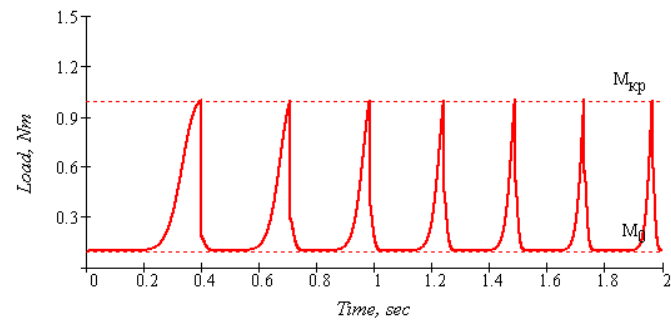

Fig. 6. Diagram of the change in the torque of resistance for a development cycle.

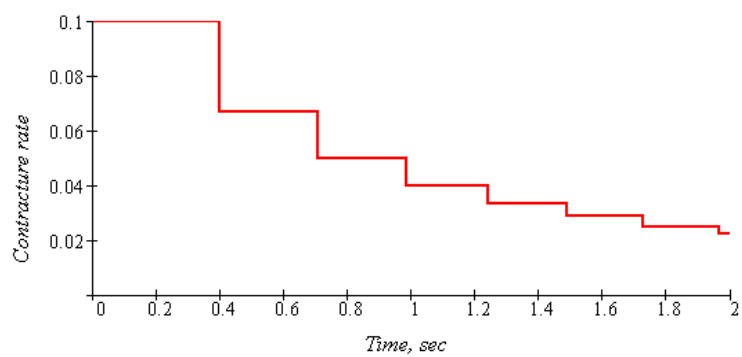

Fig. 7. Diagram of the change in the index of contracture for a development cycle.

\section{Hardware Implementation of a Microprocessor Control System of the BMT}

The developed control system was destined for the mechatronic drive of the biomechanical trainer, and consists of the following functional units: control of microcomputer, control unit by electric motor and control panel. The functional schema of the control system is illustrated in the Fig. 8 .

As operators of the microcomputer is used a single-chip 8-bit microcontroller AT89S8252, manufactured by ATMEL. The main task of the microcontroller (MC) is the engine control according to the given algorithm.

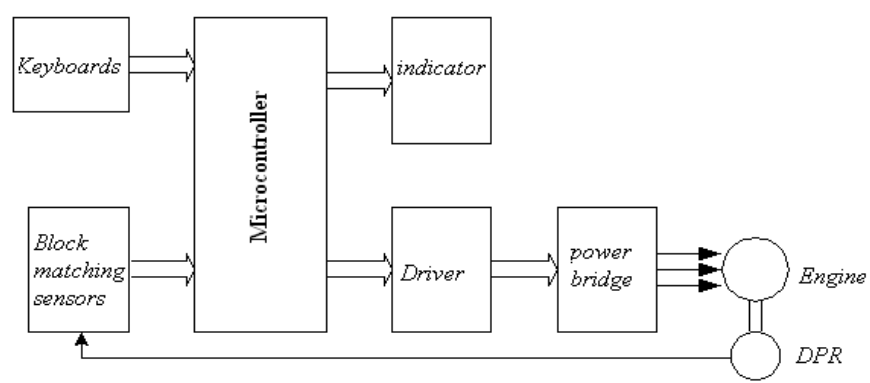

Fig. 8. Functional scheme of the control system of the biomechanical trainer. 
The drive uses a commutatorless three-phase synchronous machine, operating in a mode of ac electronic motor. For switching the windings of the motor was used a power three-phase transistor bridge CPV362M4U. The bridge link of the keys and the motor coil allows obtaining a bipolar voltage on the coil winding with a single supply. As such keys is used the powerful "Insulated-gate bipolar transistor" (IGBT). Their good current characteristics, and relatively high frequency properties allow feed the motor when during the carrier frequency of the pulse-width modulation (PWM), 16 ... $20 \mathrm{kHz}$. To match the control outputs signals of the microcontroller with the inputs of the power bridge was used the driver IR2133S. International Rectifier manufactures the driver and power bridge. The driver also execute the function of protection against current overload. As the current sensor is used the shunt R14. During an overcurrent above the permissible, the driver closes the transistor keys and switches off the engine, and also provides information about the state of emergency in the MC.

It is necessary to implement the switching of the windings of the motor in the sequence determined by the position of the permanent magnets of the rotor relative to the stator phase windings. This information is taken off by three rotor position sensors (RPS) from the phases $\mathrm{U}, \mathrm{V}, \mathrm{W}$, formed on the Hall sensors that were mounted directly on the electric motor. The signals from the sensors enter to the inputs of the MC P2.2, P2.1, P2.0. The MC, according to the information received from the RSP, realize the outputting of the control signals to the power bridge driver for the corresponding switching of the motor windings. The DD1 elements serve to organize an interruption program during the change of state of the RPS. This applies to liberate the MC from the constant interrogation of states of the RPS.

The change of the direction of rotation of the motor is realized according to the signals from the limit switches SQ1 and SQ2. They also work in the interruption mode.

To regulate the rotation velocity of the $\mathrm{MC}$ according to the programmed algorithm generates PWM signals for the power switches.

In the beginning of the work, during the turning on of the control system it is realized a setup of hardware and software of the microcontroller (timer, input-output ports, interruption system, internal registers). Then, the control system pass to the motor control mode, and the standby reception of the command proceeds from the control panel.

The control panel consists of the keypad of 16 keys (10 digital and 6 function) and a two-line alphanumeric LCD DV-16252. On the display appears the information about the operating modes of the device, values of the control parameters: $М \kappa p-$ is the threshold value of the torque of the resistance, that characterizes the onset of contractures, $N \cdot m ; M-$ is the number of cycles of development during the session of development; $N$ - the number of oscillations during the development cycle; Тосц - is the half-cycle of an oscillation, s; $\alpha$ - is the amplitude of oscillation, degree. Using the keyboard, it is possible to change these parameters.

\section{References}

[1] V.V. Morozov, A.B. Kosterin, E.A. Novikova. Smooth of dynamic links of the electromechanical actuators (monograph) VlSU, Vladimir, 1999. 158 p. (in Russian)

[2] V.V. Morozov Roller screw mechanisms. Kinematic Characteristics. (monograph)/ ViSU: Vladimir, 2005. - 78 p - ISBN 5-89368-634-9 (in Russian)

[3] Zhdanov A.V. Directions of development of mechatronic units for LVAD and TAH systems pulsating type [Text] // Mechatronics, Automation, Control / Moscow: New technologies, 2011. № 8. - pp. 51-54. ISSN 1684-6427 (in Russian)

[4] Morozov V.V., Zhdanov A.V., Kosterin A.B. Biotechnical simulators based on the mechatronic unit for the development of post-traumatic contractures [Text] // Mechatronics, Automation, Control / Moscow: New technologies, 2008. - № 3. - pp. 43-46. ISSN 1584-6427(in Russian)

[5] Morozov V.V., Zhdanov A.V., Tourscov A.I. Development of control system mechatronic modules for medical purposes [Text] // Biomedical electronics / Moscow: Radio, 2010. - №7. - P. 4-8. ISSN 1560-4136. (in Russian) 